\title{
DISTRIBUCIÓN ESPACIAL Y TENDENCIAS DE INDICADORES AGROCLIMÁTICOS EN LA ESPAÑA PENINSULAR
}

\author{
Roberto Serrano-Notivoli \\ Departamento de Geografía, Universidad Autónoma de Madrid, \\ Francisco Tomás y Valiente 1, 28049, Madrid \\ roberto.serrano@uam.es \\ Santiago Beguería \\ Estación Experimental de Aula Dei, Consejo Superior de Investigaciones \\ Científicas (EEAD-CSIC), Avda. Montañana 1005, 50059, Zaragoza \\ santiago.begueria@csic.es
}

\begin{abstract}
Resumen: Se presenta una nueva base de datos de 12 indicadores agroclimáticos para la España peninsular sobre una malla de $5 \times 5 \mathrm{~km}$ para el periodo 1981-2010. El cálculo de los indicadores se basó en la información de temperaturas máxima y mínima diarias extraídas de la base de datos STEAD. Se cartografían y describen los valores normales de todos los indicadores y se analizan los cambios durante todo el periodo mediante un análisis de tendencias. Los resultados muestran una elevada dependencia de los factores geográficos locales (elevación, latitud y distancia a la costa) en la distribución espacial de los valores normales. Sin embargo, esta influencia queda relegada en las tendencias a la costa mediterránea y las zonas montañosas. Se advierte un notable adelanto en la fecha de las primeras y últimas heladas acompañado de un descenso en el número de eventos de helada, con elevada variabilidad espacial en las tendencias. Los indicadores de integrales térmicas presentan un aumento significativo como consecuencia del incremento de las temperaturas, especialmente en primavera y verano.
\end{abstract}

Palabras clave: Agroclimatología, temperatura, climatología, tendencias, España.

Recibido: 26-02-2021. Aceptado: 10-05.2021. 


\title{
Spatial distribution and trends of agroclimatic indicators in main- land Spain
}

\begin{abstract}
A new dataset of 12 agroclimatic indicators calculated over a $5 \times 5$ km grid in 1981-2010 period is presented for peninsular Spain. The calculation of the indicators was based on the information of maximum and minimum temperatures obtained from the STEAD dataset. Normal values were mapped for all the indicators and the spatial distribution of the temporal changes was described through a trend analysis. Results showed a wide dependence from the geographical factors (elevation, latitude and distance to the coast) in the spatial distribution of the normal values. However, this influence was minimized in the trends to the Mediterranean coast and mountainous areas. A remarkable advance in the first and last frost dates was noticed alongside a decrease in the number of the events, with a high spatial variability. Those indicators of thermal sums showed a significant increase as a consequence of the raising temperatures in spring and summer.
\end{abstract}

Keywords: Agroclimatology, temperature, climatology, trends, Spain.

\section{Introducción}

La superficie agraria útil de España representa el 45.8\% de la superficie total del país. De ésta, el 76\% corresponde a cultivo de secano y el 24\% a regadío (INE, 2020). La actividad agraria es crucial para la gestión de los recursos y los medios de vida de las poblaciones rurales, y está fuertemente determinada por la variabilidad climática, que limita la disponibilidad de agua y las condiciones térmicas y, por tanto, la productividad de los cultivos. En las últimas décadas se han producido cambios en la frecuencia, duración e intensidad de los eventos climáticos extremos en España (Brunet et al., 2007, Serrano-Notivoli et al., 2018). Esta tendencia, combinada con los cambios esperados en la variabilidad de las precipitaciones y el incremento de las temperaturas medias ligado al calentamiento global (Giorgi y Lionello, 2008), cabe esperar que tenga un impacto sobre los sistemas agrícolas. Estos impactos deben ser tenidos en cuenta en futuros procesos de decisión a todos los niveles administrativos, pero también en las decisiones del día a día de los productores (Robert et al., 2016).

Los cultivos tienen una elevada sensibilidad a las condiciones climáticas durante el periodo de crecimiento y maduración. Las desviaciones con respecto a las condiciones climáticas normales, como pueden ser temperaturas más frías o cálidas de lo habitual, pueden ser responsables de grandes pérdidas en las cosechas si se dan durante momentos clave del desarrollo fenológico de los cultivos. En general, los impactos se producen como consecuencia de la ocurrencia de fenómenos extremos, definidos como 
desviaciones importantes con respecto al estado medio del clima. Sin embargo, algunos fenómenos no extraordinarios también producen afecciones sobre los cultivos. Por ejemplo, las heladas tardías son recurrentes en algunas áreas donde los agricultores prevén las pérdidas asegurando las cosechas anualmente (Hernández Navarro, 1995). Para determinar mejor la influencia del clima sobre los cultivos se han desarrollado una serie de índices agroclimáticos, que son variables derivadas de los parámetros climáticos primarios como precipitación acumulada o temperatura media del aire. El análisis estadístico de los estos índices facilita evaluar el carácter más o menos anómalo del clima a lo largo de un ciclo de cultivo, y permite establecer umbrales de anomalías que puedan afectar a su desarrollo. Estos umbrales, por supuesto, pueden variar en función de la especie o incluso la variedad utilizada, así como de otros factores como las características del suelo o incluso las técnicas de cultivo. Sin embargo, las anomalías en los índices agroclimáticos son en general representativas de situaciones adversas para los diferentes sistemas agrícolas. Algunos trabajos han tratado de identificar umbrales específicos para cultivos concretos (Tabuenca, 1983, Rötter et al., 2012, Trnka et al., 2014, Bois et al., 2014), pero en España, todavía no existe una caracterización general de los condicionantes climáticas para la agricultura, ni en valores medios ni en extremos, a pesar del enorme interés que esta caracterización puede tener. Por ejemplo, y entre otros usos, para: 1) la identificación y evaluación de zonas aptas y de riesgo para determinados cultivos; 2) la delimitación de zonas de expansión de variedades alóctonas; 3) la monitorización en tiempo real de las condiciones agroclimáticas como herramienta de decisión en las etapas de desarrollo de los cultivos o para la planificación de precios y la regulación del mercado de materias primas agrícolas; o 4) la caracterización general de la aptitud del territorio para acoger nuevas variedades de cultivo o ampliar las ya existentes. A todo esto, se añade el interés por evaluar las posibles consecuencias del cambio climático sobre la actividad agrícola.

La evaluación de los riesgos climáticos a los cultivos debe ser abordada desde una perspectiva estadística adecuada, y desde escalas y resoluciones apropiadas para este propósito. A ello se añade la necesidad de utilizar información climática fiable y continua en el espacio y en el tiempo. El uso de bases de datos en rejilla, representando las variables climáticas en una malla regular de alta resolución espacial y temporal, permite el cálculo de diversos índices agroclimáticos para todo el territorio. En España hay disponibles varias bases de datos de variables climáticas en rejilla que, a diferentes resoluciones espaciales y temporales, ofrecen información basada en un número de observatorios variable en el tiempo. La más reciente es STEAD (Spanish Temperature at Daily Scale), una base de datos de temperaturas máxima y mínima diarias sobre una malla de $5 \times 5 \mathrm{~km}$ que cubre la totalidad del territorio español para el periodo 1901-2014 (Serrano-Notivoli et al., 2019). Esta base de datos se ha elaborado a partir de la información de más de 5.000 observatorios, con longitudes de registro variables, y gracias a su elevada resolución espacial y temporal refleja adecuadamente la gran variabilidad climática española, especialmente en la península. 
Este trabajo presenta una nueva base de datos de 12 índices agroclimáticos calculados para el territorio de la España peninsular, con potencial aplicación para la planificación territorial de los sistemas agrícolas. Los índices se han calculado a escala anual para el periodo 1981-2010, sobre una malla de $5 \times 5 \mathrm{~km}$. Como objetivo secundario, también se describen los cambios temporales producidos durante el mismo periodo, mediante el análisis de la distribución espacial de las tendencias (signo, magnitud y significación) de cada uno de los indicadores. Los resultados se presentan en forma cartográfica para facilitar la interpretación, y la base de datos completa puede consultarse y descargarse libremente de forma online en la dirección https://agroclima.csic.es. Asimismo, la descripción técnica y los archivos individuales se ofrecen en acceso abierto en http://hdl.handle.net/10261/230788.

\section{Materiales y Métodos}

Utilizando la base de datos de temperaturas diarias máximas y mínimas en España STEAD (Serrano-Notivoli et al., 2019), se calcularon 12 indicadores agroclimáticos descritos en la Tabla 1 para la España peninsular en el periodo 1981-2010. A pesar de que la base de datos original cubre un periodo temporal más largo, se decidió usar este periodo por su coincidencia con el último periodo oficial de valores normales de la Organización Meteorológica Mundial. Además, el periodo seleccionado es el que cuenta con una mayor densidad de estaciones a la hora de calcular la malla, mientras que los cambios en el número de estaciones son relativamente poco importantes. Para el cálculo de los índices agroclimáticos se utilizó el paquete de R agroclim (Serrano-Notivoli et al., 2020).

Los indicadores se dividieron en dos grupos en función de su potencial aplicación en el análisis de la adaptación general de los cultivos, o por su interés para algunos cultivos concretos. El primer grupo incluye aquellos índices relacionados con la ocurrencia de heladas $\left(\mathrm{FD}_{\text {first }}, \mathrm{FD}_{\text {last, }}, \mathrm{FD}_{\text {num }}\right.$ ), factor limitante para la mayor parte de los cultivos en su periodo de desarrollo. Las heladas se consideran desde el 1 de julio hasta el 30 de junio del año siguiente. El primer y último día de helada son indicativos del periodo de exposición del cultivo, mientras que la frecuencia indica la peligrosidad, configurando así una visión completa del riesgo, siempre y cuando se complementen con la vulnerabilidad de la planta, representada por su resistencia a las bajas temperaturas.

Los índices de temperatura de los meses más cálido $\left(\mathrm{TM}_{\text {warm }}\right)$ y frío $\left(\mathrm{TM}_{\text {cold }}\right)$ son indicativos de los límites del régimen térmico a los que el cultivo ha de adaptarse, y se utilizan habitualmente para determinar el grado de idoneidad climática para cultivos específicos. A su vez, la temperatura media en la temporada de crecimiento (GST) se calcula evitando los meses más cálidos, siendo también un indicador de idoneidad. 
Tabla 1. Lista de indicadores agroclimáticos.

Se utilizan los mismos acrónimos que en Serrano-Notivoli et al. (2020)

\begin{tabular}{|c|c|c|c|}
\hline Acrónimo & Indicador & Descripción & Interés agronómico \\
\hline \multicolumn{4}{|c|}{ Adaptación general } \\
\hline $\begin{array}{l}\mathrm{FD}_{\text {first }}, \\
\mathrm{FD}_{\text {last }} \\
\mathrm{FD}_{\text {num }}\end{array}$ & $\begin{array}{l}\text { Primer día, } \\
\text { último día y } \\
\text { número de } \\
\text { heladas }\end{array}$ & $\begin{array}{l}\text { Primer y último día del año } \\
\text { y número de eventos anuales } \\
\text { en el que Tmin }<O{ }^{\circ} C \\
\text { (desde } 01 \text {-jul hasta } 30 \text {-jun) }\end{array}$ & $\begin{array}{l}\text { Impactos por congelación } \\
\text { de la planta en fases clave } \\
\text { del desarrollo }\end{array}$ \\
\hline GST & $\begin{array}{l}\text { Temperatura } \\
\text { media en la } \\
\text { temporada } \\
\text { crecimiento }\end{array}$ & $\begin{array}{l}\text { Temperatura media desde } \\
\text { 30-sep hasta } 01 \text {-abr. }\end{array}$ & \multirow{3}{*}{$\begin{array}{l}\text { Establecen los límites donde } \\
\text { los cultivos pueden } \\
\text { desarrollarse }\end{array}$} \\
\hline $\mathrm{TM}_{\text {cold }}$ & $\begin{array}{l}\text { Temperatura } \\
\text { del mes } \\
\text { más frío }\end{array}$ & $\begin{array}{l}\text { Valor mínimo de la } \\
\text { temperatura de los } \\
\text { meses del año }\end{array}$ & \\
\hline $\mathrm{TM}_{\text {warm }}$ & $\begin{array}{l}\text { Temperatura } \\
\text { del mes } \\
\text { más cálido }\end{array}$ & $\begin{array}{l}\text { Valor máximo de la } \\
\text { temperatura de los } \\
\text { meses del año }\end{array}$ & \\
\hline \multicolumn{4}{|c|}{ Específicos para cultivos } \\
\hline $\begin{array}{l}\text { EHE }_{\text {day }} \\
\text { EHE }_{\text {num }}\end{array}$ & $\begin{array}{l}\text { Primer día y } \\
\text { frecuencia de } \\
\text { exposición a } \\
\text { calor extremo }\end{array}$ & $\begin{array}{l}\text { Primer día del año y número } \\
\text { de eventos anuales en los que } \\
\text { Tmax }>35^{\circ} \mathrm{C} \text { durante al menos } \\
3 \text { días en un periodo de } 5 \\
\text { consecutivos después de la } \\
\text { floración (31-may) y } \\
\text { hasta la madurez (31-jul) }\end{array}$ & \multirow[t]{2}{*}{$\begin{array}{l}\text { Impactos por altas } \\
\text { temperaturas de la planta } \\
\text { en fases clave del } \\
\text { desarrollo. De especial } \\
\text { interés para el cereal }\end{array}$} \\
\hline $\mathrm{T} 35_{\text {first }}$ & $\begin{array}{l}\text { Primer día } \\
\operatorname{Tmax}>35^{\circ} \mathrm{C}\end{array}$ & $\begin{array}{l}\text { Primer día del año en el } \\
\text { que Tmax }>35^{\circ} \mathrm{C} \\
\text { (desde } 1 \text {-ene hasta } 1 \text {-sep) }\end{array}$ & \\
\hline GDD & $\begin{array}{l}\text { Growing } \\
\text { degree days }\end{array}$ & $\begin{array}{l}\text { Suma térmica diaria de las } \\
\text { temperaturas medias } \\
\text { superiores a } 10{ }^{\circ} \mathrm{C} \\
\text { (desde } 30 \text {-sep hasta } 01 \text {-abr) }\end{array}$ & $\begin{array}{l}\text { Adaptabilidad de los } \\
\text { cultivos a las fechas de la } \\
\text { temporada de crecimiento }\end{array}$ \\
\hline $\mathrm{HI}$ & Huglin Index & $\begin{array}{l}\text { Suma térmica diaria de las } \\
\text { temperaturas medias } \\
\text { superiores a } 10^{\circ} \mathrm{C} \\
\text { (desde } 01 \text {-abr hasta } 30-\text { sep) } \\
\text { con una corrección latitudinal }\end{array}$ & \multirow{2}{*}{$\begin{array}{l}\text { Viñedo: adaptación de las } \\
\text { variedades de uva a los } \\
\text { regímenes térmicos }\end{array}$} \\
\hline BEDD & \begin{tabular}{l|} 
Biologically \\
Effective \\
Degree-Days
\end{tabular} & $\begin{array}{l}\text { Suma térmica diaria de las } \\
\text { temperaturas medias desde } \\
10^{\circ} \mathrm{C} \text { hasta } 19^{\circ} \mathrm{C} \\
\text { (desde } 01 \text {-abr hasta } 30-\text { sep) } \\
\text { con una corrección latitudinal }\end{array}$ & \\
\hline
\end{tabular}


El segundo conjunto de indicadores se calculó con el objetivo de servir de análisis para cultivos específicos, aunque también informan acerca de la ocurrencia de eventos extremos que pueden afectar a otros cultivos en general. Por un lado, la exposición a calor extremo (EHE) define situaciones de persistencia de altas temperaturas utilizando un umbral de $35{ }^{\circ} \mathrm{C}$ que, aunque es potencialmente alto para el ciclo vegetativo de diversos cultivos que con temperaturas inferiores ya se pueden ver afectados, está pensado principalmente para aquellos en los que sea ha demostrado una incidencia como es el caso del trigo y la cebada (Martí, 1991; Contreras-Moreira et al., 2019), el arroz o el maíz (Martí, 1993). De la misma manera que con las heladas, tanto el primer día de ocurrencia (T35 first) como la frecuencia de estos eventos, ayudan a determinar la situación de riesgo.

Los indicadores GDD, HI y BEDD son diferentes versiones de integrales térmicas a partir de la temperatura diaria, y son de utilidad para evaluar la aptitud de un territorio respecto a las diferentes variedades vitícolas (Jones et al., 2010; Hall y Jones, 2010). La variable GDD, no obstante, es ampliamente utilizada en otros cultivos (Ahmad et al., 2017), e incluso con especies no agrícolas (Maclean et al., 2017).

Con el fin de evaluar los cambios producidos durante el periodo 1981-2010, se calcularon las tendencias de las medianas anuales de todos los índices, para todas las celdas del área de estudio. Se utilizó la prueba de Mann-Kendall (Mann, 1945) para evaluar el signo y la significación de la tendencia, determinada por $=0,05$. Como algunos indicadores basados en el cálculo de extremos de temperatura no se dan todos los años en todo el territorio, los resultados en estas zonas podrían dar lugar a equívocos en la interpretación de la evolución temporal cuando se comparan con el resto del territorio, así que las series de datos incompletas se desecharon del análisis de tendencias y aparecen en blanco en los mapas.

\section{Resultados}

\subsection{Indicadores para la adaptación general de los cultivos}

En general, la distribución espacial de los índices agroclimáticos muestra una relación clara con la elevación (Figura 1), debido a que están calculados exclusivamente con datos de temperatura máxima y mínima. No obstante, existen variaciones espaciales definidas por la continentalidad, la homogeneidad orográfica y la proximidad a la costa, que configuran la potencialidad de cultivo de determinadas áreas.

La fecha más frecuente para la primera helada $\left(\mathrm{FD}_{\text {first }}\right)$ varía desde principios de julio en las zonas más elevadas de los Pirineos centrales y Cordillera Cantábrica hasta la tercera semana de marzo al sur de la costa mediterránea. En este rango se produce un claro gradiente altitudinal que tiene su umbral intermedio hacia mediados de noviem- 


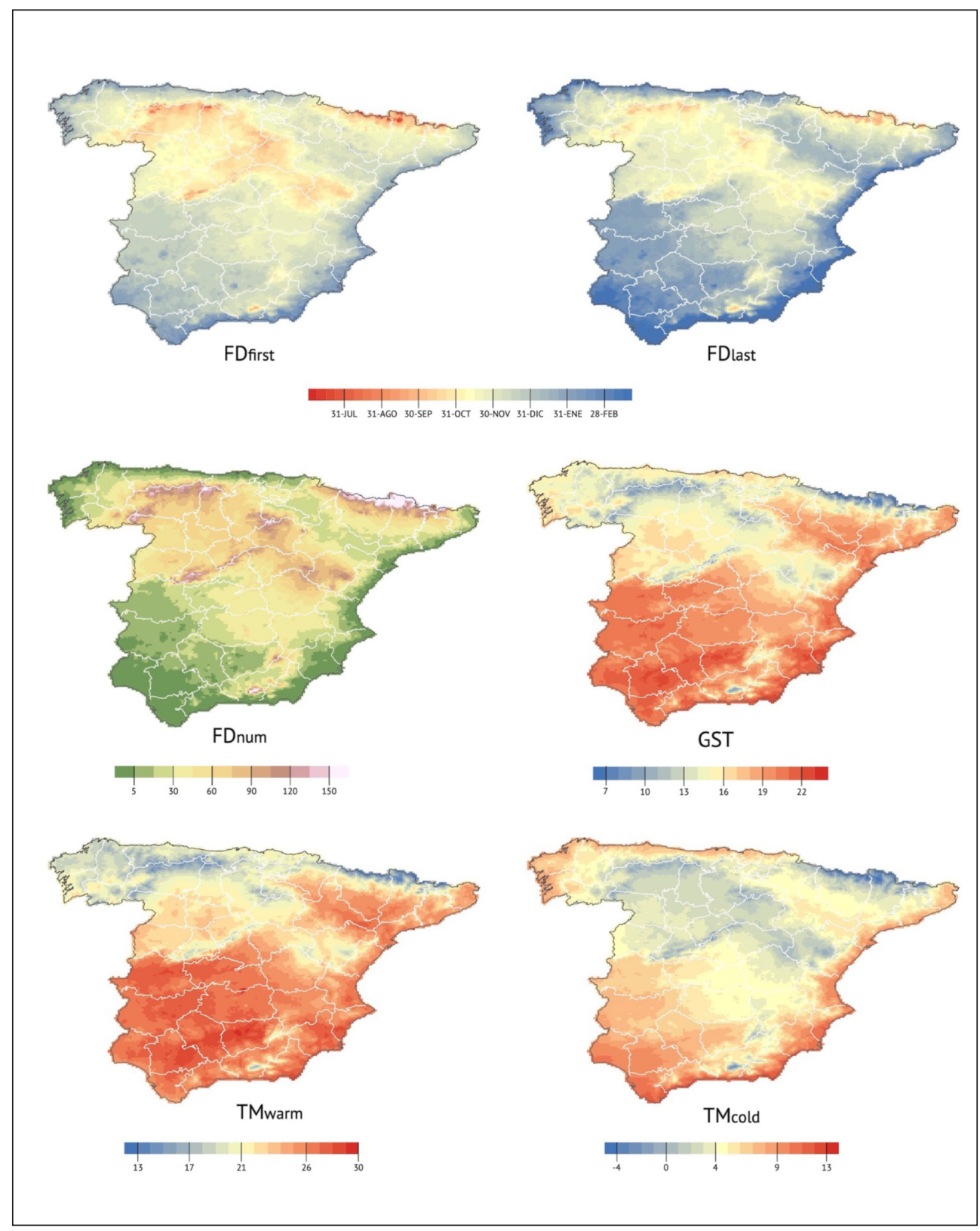

Figura 1. Distribución espacial de la mediana de los valores de indicadores agroclimáticos de adaptación general en el periodo 1981-2010.

Fuente: STEAD (Serrano-Notivoli et al., 2019). Elaboración propia. 
bre en altitudes entre 600 y 700 m.s.n.m. La fecha de la última helada $\left(\mathrm{FD}_{\text {last }}\right)$ sigue un patrón similar, aunque en toda la costa mediterránea y especialmente en el sur las heladas terminan muy pronto, hacia mediados de marzo. El número mediano de días de helada $\left(\mathrm{FD}_{\text {num }}\right)$ muestra un patrón similar, pero con menor variabilidad en la mitad sur peninsular, la costa mediterránea y Galicia, donde se dan los valores más bajos, por debajo de 5 días de helada al año en las zonas costeras. Es en aquellas áreas con elevaciones por encima de 400 metros donde la frecuencia se incrementa hasta superar los 30 días por año, con una progresión casi lineal con la altitud hasta llegar a más de 150 días por año en todas las zonas por encima de 2000 m.s.n.m. en los Pirineos, Cordillera Cantábrica, Montes de León, Gredos y Peñalara y Picos de Urbión. Por debajo de los $40 \stackrel{\circ}{N}$ de latitud solo Sierra Nevada alcanza esa cifra. En general, las heladas pueden extenderse desde menos de un mes en las zonas costeras con ocurrencias anuales de menos de una semana en total, hasta periodos de 8 y 9 meses de los que al menos 3 de ellos contienen días de helada. En la zona intermedia, lo habitual en las dos mesetas y los piedemontes de las grandes cordilleras son periodos de heladas desde principios del otoño hasta finales del invierno, con ocurrencias fuertemente dependientes de la elevación, que pueden variar desde los 30 hasta los 90 días por año.

Los indicadores más genéricos de idoneidad climática para los cultivos se definen por los regímenes térmicos promedio. Estos reflejan, como no puede ser de otra manera, los factores geográficos mencionados anteriormente de altitud y continentalidad. Por un lado, la temperatura media de la estación de crecimiento (GST) muestra una clara diferencia entre la cálida mitad sur peninsular, con temperaturas medias superiores a $17^{\circ} \mathrm{C}$ (exceptuando las áreas elevadas de Sierra Nevada y Cazorla) y la fría mitad norte por debajo de ese umbral, llegando a temperaturas medias inferiores a 10 grados (límite inferior de acumulación de grados día para cultivos de cereal y vitícolas, entre otros) en las áreas por encima de 1.000 m.s.n.m. El mapa de la temperatura media del mes más cálido $\left(\mathrm{TM}_{\text {warm }}\right)$ muestra un patrón muy similar, puesto que todo el territorio tiene su periodo más cálido durante la canícula, en julio o agosto. La temperatura del mes más frío $\left(\mathrm{TM}_{\text {cold }}\right)$, por otra parte, muestra un patrón más parecido al de las fechas de heladas, siendo en prácticamente todo el territorio el mes de enero el más frío.

\subsection{Indicadores específicos para cultivos}

Aunque los indicadores específicos para determinados cultivos también muestran un patrón espacial que puede encajar con la distribución altitudinal, éste no es tan claro como en el conjunto anterior de indicadores (Figura 2).

La fecha del primer día de ocurrencia de eventos de exposición a calor extremo $\left(\mathrm{EHE}_{\mathrm{day}}\right)$ es más temprana, en torno a principios de final de mayo en la mitad sur peninsular y en el valle del Ebro. Esta fecha se va posponiendo hasta final de julio e incluso más tarde en la meseta norte, la Cordillera Ibérica y todo el norte peninsular ex- 


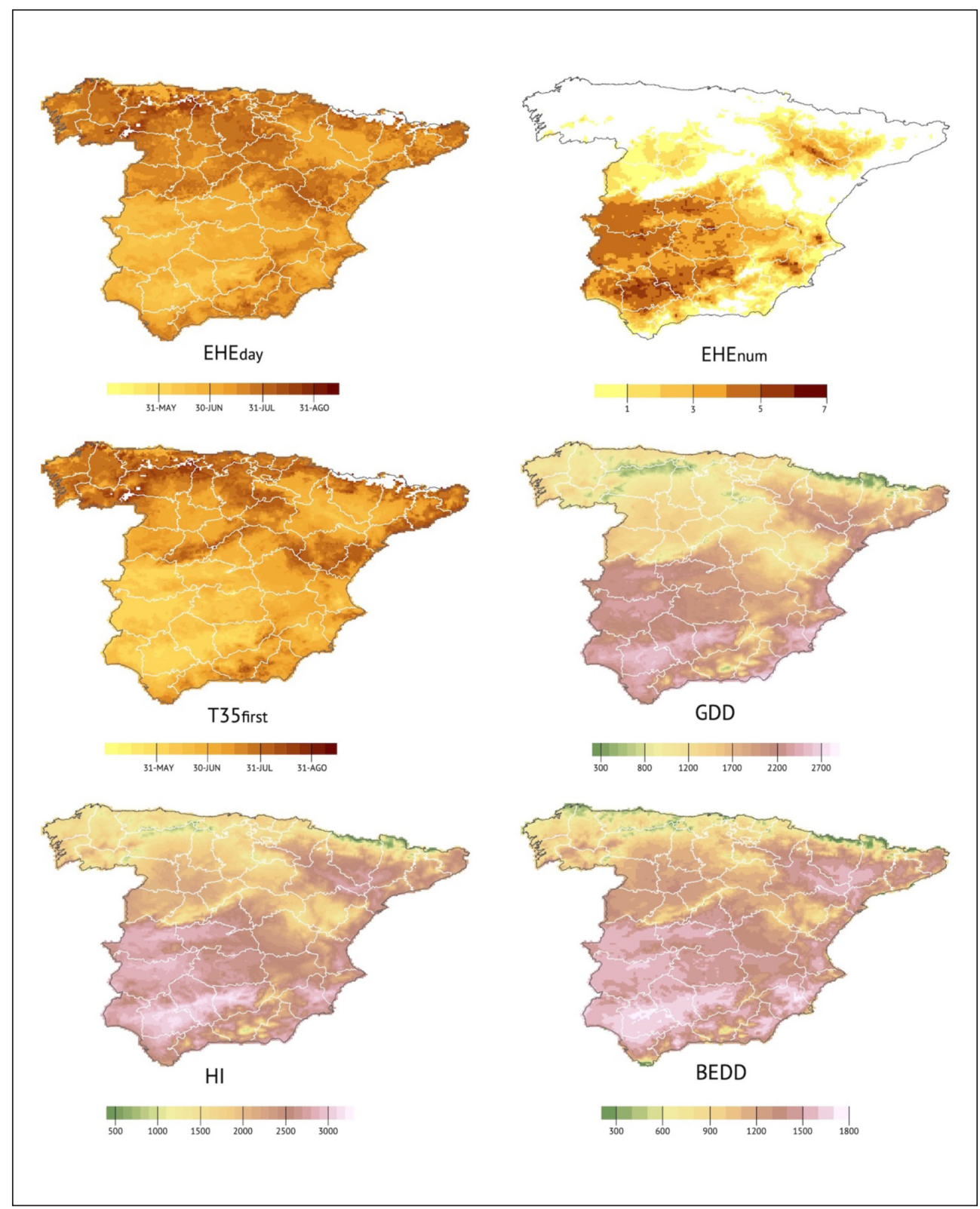

Figura 2. Distribución espacial de la mediana de los valores de indicadores agroclimáticos específicos para cultivos en el periodo 1981-2010.

Se indican en blanco las áreas con sin ocurrencia del evento.

Fuente: STEAD (Serrano-Notivoli et al., 2019). Elaboración propia. 
cepto la costa cántabra. También las zonas elevadas de la costa mediterránea alcanzan fechas cercanas a mediados de agosto. Esto indica que tanto la altitud como la cercanía a la costa ejercen un factor suavizador de los eventos de altas temperaturas, favoreciendo la idoneidad de estas áreas para ciertos cultivos. Hay que tener en cuenta que en estas zonas, y especialmente en el norte peninsular, estos eventos no son habituales, lo cual influye en la fiabilidad de la fecha de inicio. De hecho, el número mediano de eventos de calor extremo $\left(\mathrm{EHE}_{\text {num }}\right)$ es cero en gran parte de estas áreas, y completamente inexistente en las zonas más elevadas de los Pirineos y la Cordillera Cantábrica. La mayor frecuencia de eventos de calor extremo se da en el valle del Guadalquivir y del Segura en menor medida, aunque se observan valores superiores a 4 eventos por año en los sectores deprimidos del Tajo y el Guadiana. La fecha del primer día con temperaturas superiores a $35{ }^{\circ} \mathrm{C}\left(\mathrm{T} 35_{\text {first }}\right)$ coincide en su distribución espacial con el de la fecha de inicio de los eventos de calor extremo, ya que ambos índices utilizan el mismo umbral térmico.

Los índices agronómicos específicos para los cultivos vitícolas, como los Growing Degree Days (GDD), el índice helio-térmico de Huglin (HI) o el Biologically Effective Degree-Days (BEDD) son muy similares en su patrón espacial. Así, las acumulaciones térmicas más elevadas $\left(>1500^{\circ} \mathrm{C}\right)$ se dan en el sur peninsular, especialmente en los valles del Guadalquivir, Guadiana, Tajo y Segura, y en menor medida en el norte en el valle del Ebro. El resto del territorio mantiene una clara dependencia de la altitud, aunque los dos últimos indicadores (HI y BEDD) aseguran una mayor acumulación de grados-día en la meseta norte y el valle del Ebro, ya que ponderan positivamente el factor latitudinal.

\subsection{Tendencias en indicadores para la adaptación general de los cultivos}

Las tendencias en las fechas de inicio de las heladas $\left(\mathrm{FD}_{\text {first }}\right)$ muestran una tendencia negativa significativa (es decir, adelanto en la fecha la primera helada) en la meseta norte, las estribaciones occidentales de la Cordillera Ibérica, el Sistema Bético y en zonas aisladas de la meseta sur (Figura 3). Se produce un retraso generalizado (tendencia positiva del índice) en el norte peninsular, pero con escasas zonas donde éste es significativo. El adelanto en la fecha de la última helada (FDlast) es significativo en los piedemontes de la mitad norte peninsular, la Cordillera Ibérica y el sector oriental de la meseta sur. Se observa un comportamiento inverso, es decir un retraso en la fecha de la última helada, en la costa mediterránea meridional, pero no significativo, y en la confluencia de los ríos Cinca y Segre, en el noreste peninsular, posiblemente debido a la alta frecuencia de nieblas. Las tendencias en el número de días de helada $\left(\mathrm{FD}_{\text {num }}\right)$ son muy variables, pero se observa una cierta predominancia de la reducción del número de días de helada en la mitad occidental de la península, con una concentración generalizada en la Cordillera Cantábrica, y también en algunas zonas elevadas de la Ibérica, Cataluña y el sector montañoso de la costa mediterránea en general. 


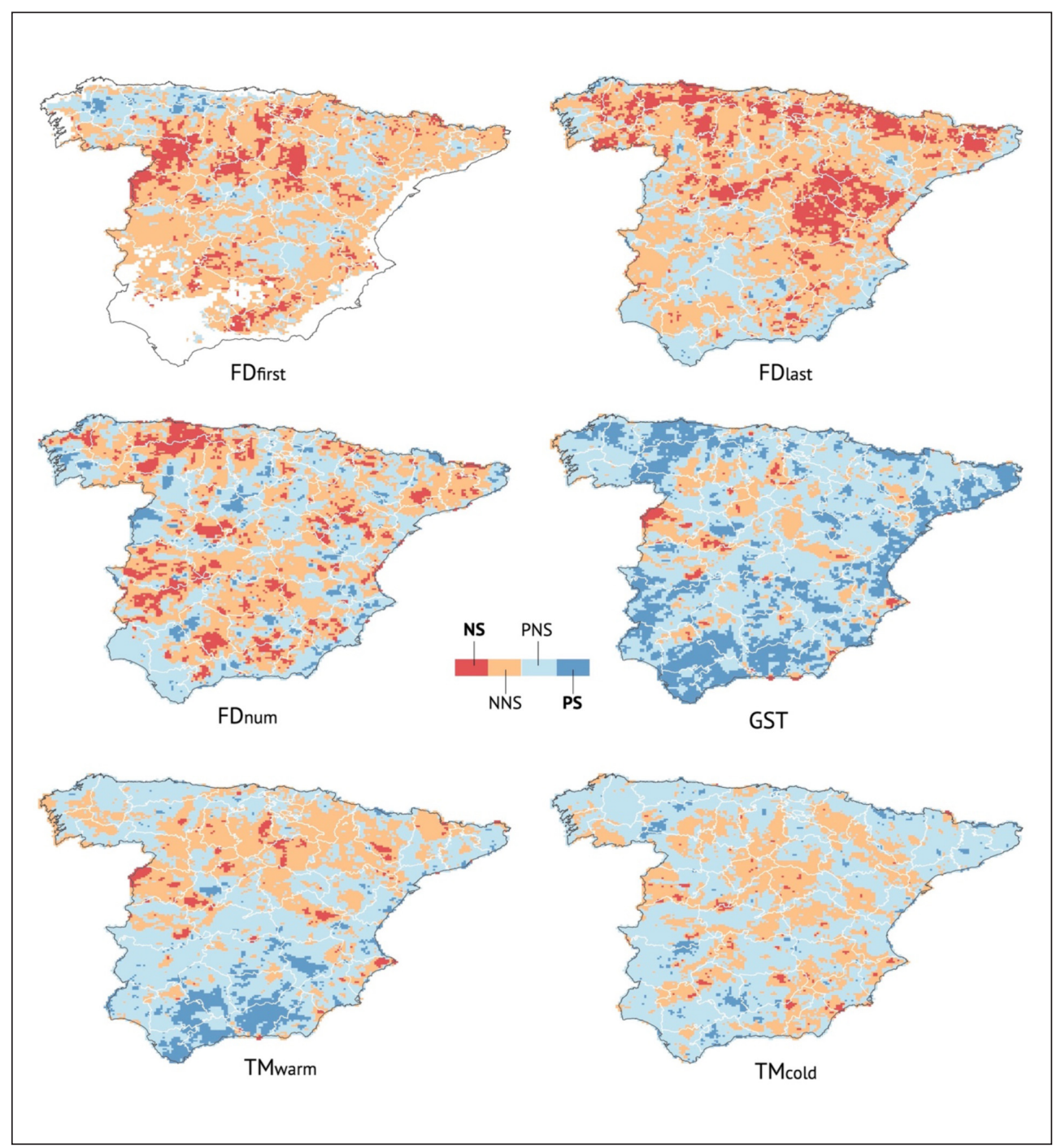

Figura 3. Tendencias de los valores de indicadores agroclimáticos de adaptación general en el periodo 1981-2010. NS: negativo significativo, NNS: negativo no significativo, PNS: positivo no significativo, PS: positivo significativo. 
Las temperaturas en los tres indicadores sobre la potencialidad de cultivo en el territorio muestran una tendencia general positiva, aunque no significativa en todos los casos. Considerando la temporada más cálida (GST y TM warm ), se observa un incremento de las temperaturas (tendencia positiva) especialmente en la mitad sur peninsular y en las zonas costeras (excepto Alicante, que muestra el comportamiento inverso). Las tendencias en las temperaturas mínimas $\left(\mathrm{TM}_{\mathrm{cold}}\right)$ son en general no significativas, pero con una cierta predominancia de las de signo positivo en prácticamente todo el territorio excepto las zonas de interior.

\subsection{Tendencias en indicadores específicos para cultivos}

Las tendencias en los eventos de calor extremo (restringidas a las áreas con ocurrencias anuales durante todo el periodo de estudio) muestra un claro adelanto en la fecha de ocurrencia $\left(\mathrm{EHE}_{\text {day }}\right.$ ), significativo en la cuenca del Tajo y del Guadalquivir, y un incremento en el número de eventos por año $\left(\mathrm{EHE}_{\text {num }}\right)$, significativo en todo el valle del Guadalquivir (Figura 4). La fecha del primer día con temperaturas superiores a $35^{\circ} \mathrm{C}\left(\mathrm{T} 35_{\text {first }}\right)$ también muestra un adelanto generalizado, pero las zonas de tendencia significativa son menos frecuentes y más dispersas en el territorio. Con todo, hay un patrón claro de adelanto en la mitad sur peninsular exceptuando las zonas montañosas.

Las tendencias de la suma térmica calculada por el método tradicional (GDD) o con el índice de Huglin (HI) muestran un patrón similar, con preponderancia de los valores positivos que son significativos en la costa mediterránea excepto en la provincia de Alicante y las costas de Almería y Málaga, y negativas significativas en la mitad sur de la meseta norte y también en el sector más elevado de la Sierra de Gredos. Estos patrones son diferentes en el indicador BEDD, en el que la suma térmica se limita con un umbral superior de $19{ }^{\circ} \mathrm{C}$. Este indicador muestra una tenencia negativa significativa en el sur a lo largo de toda la costa mediterránea andaluza y de las provincias de Alicante y Valencia, en el norte en la costa cántabra, asturiana y en la provincia de Vizcaya, y en las estribaciones occidentales del Sistema Central y los Montes de Toledo. Por otro lado, muestra una tendencia positiva en el noreste peninsular, siendo significativa en el extremo noreste de Cataluña y los Pirineos aragoneses. 


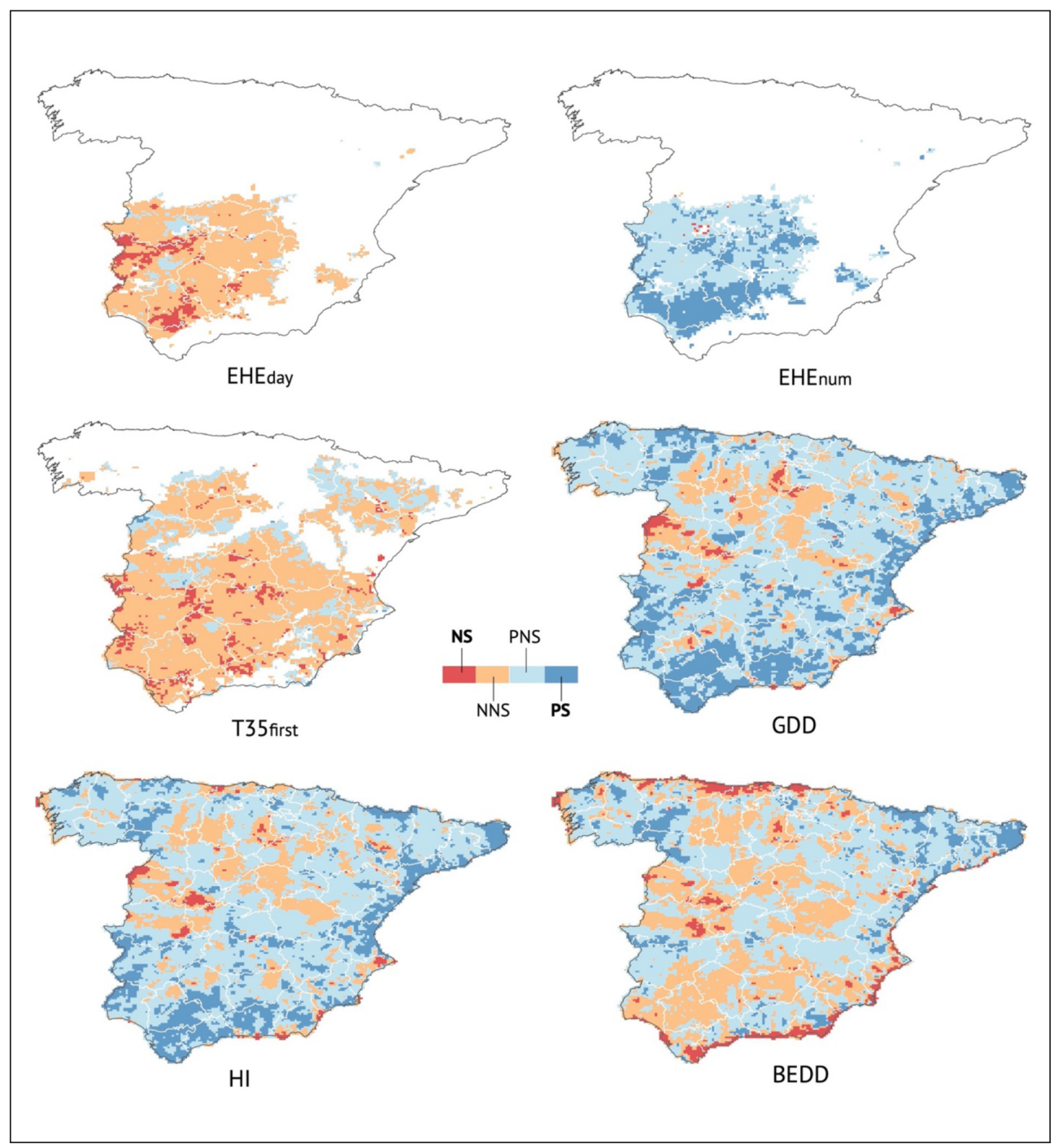

Figura 4. Tendencias de los valores de indicadores agroclimáticos específicos para cultivos en el periodo 1981-2010. NS: negativo significativo, NNS: negativo no significativo, PNS: positivo no significativo, PS: positivo significativo.

Fuente: STEAD (Serrano-Notivoli et al., 2019). Elaboración propia. 


\section{Discusión y conclusiones}

Los 12 índices agroclimáticos analizados muestran tendencias no significativas en la mayor parte del territorio peninsular español (72,8\%, Tabla 2$)$, con una variabilidad espacial elevada. De la misma manera que ocurre con la precipitación, la configuración orográfica y latitudinal son dos de los factores geográficos que juegan un papel más importante en la distribución espacial de la temperatura (Serrano-Notivoli et al., 2018). En el caso de los indicadores agroclimáticos, todos ellos tienen una relación de intensidad variable con la elevación (Figura 5) pero significativa, en cualquier caso. Los indicadores de adaptación general muestran correlaciones más altas que los específicos para cultivos, aunque probablemente se debe a que los primeros se derivan de un cálculo más directo sobre las temperaturas máximas y mínimas, mientras que los segundos representan integrales térmicas o condicionantes de acumulación que tienen una mayor irregularidad interanual, por lo que la respuesta orográfica se disipa levemente. Es destacable que las correlaciones más altas (Pearson $\geq \pm 0,79$ ) se dan en los indicadores de temperaturas mínimas $\left(\mathrm{FD}_{\text {num }} \mathrm{y} \mathrm{TM}_{\text {cold }}\right.$ ), y las más bajas (Pearson £ 0,50) en los que muestran fechas de inicio de eventos de temperatura máxima $\left(\mathrm{EHE}_{\text {first }} \mathrm{y}\right.$ T35 first . No obstante, hay que destacar que la rejilla de datos de temperatura utilizada para el cálculo cubre completamente el territorio, mientras que las observaciones originales con las que se construyó no son frecuentes por encima de los 1.000 m.s.n.m. y escasas sobre los 1.500. Esto puede provocar, junto a una frecuencia no medible de ocurrencia de inversiones térmicas en altura, que las relaciones entre los índices y la elevación en cotas elevadas tenga más incertidumbre. En cualquier caso, esto no resta importancia a los resultados, pues la mayor parte de los cultivos se encuentra bajo esas cotas, donde la fiabilidad de los datos es mucho mayor.

Sin embargo, los resultados del análisis de las tendencias muestran que, a pesar de que la elevación y la latitud sí participan de manera determinante en la distribución espacial de los indicadores agroclimáticos, las tendencias están lejos de mostrar un patrón orográfico o geográfico bien marcado en todas las situaciones. Sin embargo, sí se observa un comportamiento diferenciado entre la costa mediterránea y el interior peninsular en la mayoría de los índices, aunque este patrón no es tan claro como para obtener conclusiones sólidas.

Por una parte, las cifras globales indican que las heladas comienzan y terminan antes en la temporada (tendencias negativas significativas en el 9,7 y 17,2 \% del territorio, respectivamente) y se producen con menor frecuencia (11,7\%). Este patrón de descenso en las heladas ha propiciado la aparición de nuevas zonas potenciales de cultivo para determinadas especies. Por ejemplo, aunque la variabilidad es alta, se observa una coherencia espacial en la Cordillera Ibérica turolense que se extiende hasta el sector noreste de la meseta sur. Esta zona, que alcanza habitualmente los récords de temperatura mínima de todo el país $\left(<-20^{\circ} \mathrm{C}\right)$, adelanta la fecha del fin de las heladas, 

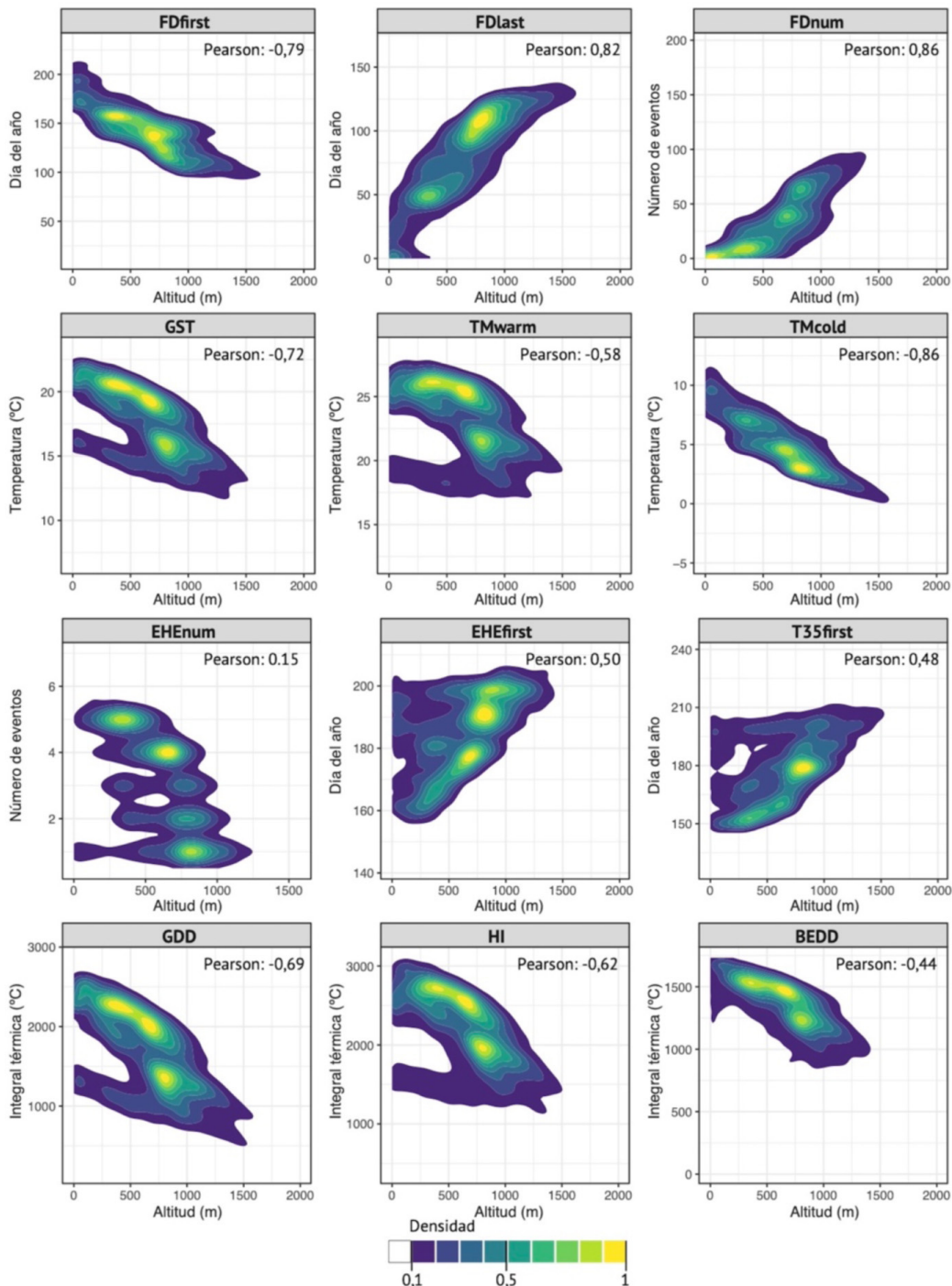

Figura 5. Relación entre los valores de los indicadores agroclimáticos (ejes Y) y la altitud (ejes X). Los colores representan de menor (azul) a mayor (amarillo) frecuencia (densidad) de celdas del grid en cada indicador. Solo se consideran las celdas por debajo de 2.000 m.s.n.m. y las densidades superiores a 0,1. Todas las correlaciones son significativas con un $p$-value $<0.01$. 
pero no aumenta tanto el número de eventos. Este hecho es consistente con el reciente trabajo de Sandonis et al. (2021), donde se muestra un incremento de las temperaturas de primavera en el mismo periodo y localización, mientras que las de invierno permanecen inalterables. Este incremento, junto a otro de menor entidad y centrado en el sector sureste peninsular, concuerda con las tendencias positivas significativas dominantes observadas en los indicadores que usan temperaturas de primavera y verano para su cálculo, como el de la temporada de crecimiento ( $28,5 \%$ del territorio), la temperatura del mes más cálido $(9,3 \%)$ o los que producen sumas térmicas (GDD: 23,7\%, HI: 19,7\%; BEDD: 9,47\%). En éstos, se observan tendencias positivas especialmente en la mitad sur peninsular. En este sentido, el indicador BEDD resalta más las diferencias, mostrando un patrón muy diferente, con tendencias negativas significativas muy claras en las zonas costeras en general, incluyendo la cantábrica. Este hecho probablemente se debe a que su cálculo tiene en cuenta un rango de temperaturas entre $10 \mathrm{y}$ $19{ }^{\circ} \mathrm{C}$, muy por debajo de las temperaturas máximas promedio, que son las que acusan un incremento mayor en el periodo 1981-2010 (Peña-Angulo et al., 2021).

Tabla 2. Porcentaje de territorio con tendencias positivas significativas (Ps), negativas significativas (Ns) o tendencias no significativas de cualquier signo (ns).

El umbral de significación se estableció en $p<0,05$. Se indican en negrita las tendencias significativas más frecuentes para cada indicador

\begin{tabular}{|l|c|c|c|}
\hline Indicador & $P s$ & $N s$ & $n s$ \\
\hline $\mathrm{FD}_{\text {first }}$ & 1,13 & $\mathbf{9 , 6 6}$ & 70,76 \\
\hline $\mathrm{FD}_{\text {last }}$ & 0,95 & $\mathbf{1 7 , 2 3}$ & 81,82 \\
\hline $\mathrm{FD}_{\text {num }}$ & 5,52 & $\mathbf{1 1 , 7}$ & 82,78 \\
\hline $\mathrm{GST}$ & $\mathbf{2 8 , 5}$ & 1,64 & 69,86 \\
\hline $\mathrm{TM}_{\text {cold }}$ & $\mathbf{3 , 0 8}$ & 1,26 & 95,96 \\
\hline $\mathrm{TM}_{\text {warm }}$ & $\mathbf{9 , 2 9}$ & 2,76 & 87,95 \\
\hline $\mathrm{EHE}_{\text {day }}$ & 0,03 & $\mathbf{2 , 9 8}$ & 23,07 \\
\hline EHE \\
\hline num & $\mathbf{9 , 9 3}$ & 0,09 & 68,86 \\
\hline T35 & $\mathbf{2 3 , 6 6}$ & 1,99 & 74,35 \\
\hline HI & 0,20 & $\mathbf{4 , 1 6}$ & 55,36 \\
\hline BEDD & $\mathbf{1 9 , 7 2}$ & 1,95 & 78,33 \\
\hline
\end{tabular}

Fuente: Elaboración propia. 
El presente estudio es el primer análisis integral de un conjunto de indicadores agroclimáticos para la España peninsular usando una base de datos de alta resolución espacial y temporal. Aunque ha habido iniciativas previas que han abordado la potencialidad de los cultivos en España en función de características climáticas, éstas se han centrado principalmente en el impacto de las sequías (Jiménez-Donaire et al., 2020, Almendra-Martín et al., 2021) o en el uso de variables climáticas combinadas para regiones o cultivos específicos (Quiroga e Iglesias, 2009, Contreras-Moreira et al., 2019, Arenas-Castro et al., 2020), y no tanto en el impacto de las temperaturas (promedio y extremas). Sí existen, en cambio, algunos ejemplos a escala regional (Rodrigo Comino et al., 2014). Por ejemplo, Resco et al. (2016) hacen un completo análisis de tres índices agroclimáticos: Huglin Index, Cool Night Index (similar a $\mathrm{TM}_{\text {cold }}$ ) y Dryness Index, para la adaptación de variedades vitícolas. Aunque la aproximación de nuestro trabajo es más general, puede servir de base para estudios de características similares, proporcionando la información de partida para un periodo climático normal (1981-2010) y abriendo la puerta a sucesivos estudios de detalle sobre regiones o cultivos concretos, o sobre periodos temporales más extendidos.

A pesar del potencial de aplicación que tiene esta nueva base de datos de índices agroclimáticos, como también la herramienta agroclim (Serrano-Notivoli et al., 2020) con la que ha sido construida, ambas tienen algunas limitaciones en cuanto a su aplicabilidad. Por ejemplo, no se consideran aquellos indicadores derivados de la precipitación o la evapotranspiración, que son claves para evaluar la adaptabilidad de los cultivos a la disponibilidad de agua, un factor de suma importancia en la España peninsular. En este contexto, el balance hídrico es posiblemente el indicador más importante, y requiere del cálculo de la evapotranspiración de referencia, que ya está disponible para el área de estudio a una resolución temporal semanal (Tomas-Burguera et al., 2019), así como la precipitación a escala diaria (Serrano-Notivoli et al., 2017). En futuras versiones de la base de datos de índices agroclimáticos se espera poder incorporar variables derivadas del uso combinado de ambas variables.

La base de datos de índices agoclimáticos para la España peninsular presentada en este trabajo representa una nueva herramienta para el estudio de los riesgos sobre los cultivos asociados a las condiciones climáticas extremas. Estos índices aportan una amplia variedad de indicadores, siendo algunos específicos para determinados cultivos y teniendo otros un carácter más general. Esta nueva base de datos constituye una fase inicial de la evaluación de los riesgos agroclimáticos en España, y los trabajos futuros tendrán que ir orientados a la especificidad de las características climáticas representadas por cada índice, para regiones y cultivos concretos. Por ejemplo, no tiene el mismo impacto una helada de varios días de duración con temperaturas cercanas a cero grados, que otra de un solo día en la que se alcancen temperaturas negativas muy extremas. Además, la incidencia de estos fenómenos sobre los cultivos dependerá también, entre otros, del tipo de cultivo, de sus características genéticas, de la presencia de infraestructuras o medidas para paliar los efectos de la temperatura, de la localización dependiente de los efectos orográficos y del tipo de clima, donde la mayor o menor frecuencia de altas o bajas temperaturas condicionará la presencia de determinados cultivos. 


\section{Agradecimientos}

R.S.N. pertenece al Grupo H38 "Clima, Agua y Cambio Global" del "Programa de grupos de investigación" del Gobierno de Aragón. Este trabajo ha contado con financiación del proyecto CGL2017-83866-C3-3-R (CLICES, Climate of the last Century in the Spanish mainland), perteneciente al Programa Estatal de I+D+i Orientada a los Retos de la Sociedad del Ministerio de Ciencia e Innovación.

\section{Bibliografía}

Ahmad, S., Abbas, Q., Abbas, G., Fatima, Z., Atique-ur-Rehman, Naz, S., Younis, H., Khan, R.J., Nasim, W., Habib ur Rehman, M., Ahmad, A., Rasul, G., Khan, M.A., Hasanuzzaman, M. (2017). Quantification of Climate Warming and Crop Management Impacts on Cotton Phenology. Plants, 6(1), 7. DOI: https://doi.org/10.3390/plants6010007

Almendra-Martín, L., Martínez-Fernández, J., González-Zamora, Á., Benito-Verdugo, P., HerreroJiménez, C.M. (2021). Agricultural Drought Trends on the Iberian Peninsula: An Analysis Using Modeled and Reanalysis Soil Moisture Products. Atmosphere, 12(2), 236. DOI: https://doi.org/ 10.3390/atmos12020236

Arenas-Castro, S., Gonçalves, J.F., Moreno, M., Villar, R. (2020). Projected climate changes are expected to decrease the suitability and production of olive varieties in southern Spain. Science of The Total Environment, 709, 136161. DOI: https://doi.org/10.1016/j.scitotenv.2019.136161.

Bois, B., Gavrilescu, C., Moriondo, M., Jones, G.V. (2014). Wine growing regions global climate analysis. En: Jones, G.V. and Doran, N. Proceedings XI International Terroir Congress 2016, pp. 30-35.

Brunet, M., Jones, P.D., Sigró, J., Saladié, O., Aguilar, E., Moberg, A., Della-Marta, P.B., Lister, D., Walther, A., López, D. (2007). Temporal and spatial temperature variability and change over Spain during 1850-2005. Journal of Geophysical Research. Atmospheres, 112(D12). DOI: https://doi.org/10.1029/2006JD008249

Contreras-Moreira, B., Serrano-Notivoli, R., Mohammed, N.H., Cantalapiedra, C.P., Beguería, S., Casas, A.M., Igartua, E. (2019). Genetic association with high resolution climate data reveals selection footprints in the genomes of barley landraces across the Iberian Peninsula. Molecular Ecology, 28(8), pp. 1-19. DOI: https://doi.org/10.1111/mec.15009

Giorgi, F., Lionello, P. (2008). Climate change projections for the Mediterranean region. Global and Planetary Change, 63(2-3), pp. 90-104. DOI: https://doi.org/10.1016/j.gloplacha. 2007.09.005.

Hall, A., Jones, G.V. (2010). Spatial analysis of climate in winegrape-growing regions in Australia. Australian Journal of Grape Wine Research, 16, pp. 389-404. DOI: https://doi.org/10. 1111/j.1755-0238.2010.00100.x

Hernández Navarro, M.L. (1993). La agroclimatología, instrumento de planificación agrícola. Geographicalia, 30, pp. 213-228. DOI: https://doi.org/10.26754/ojs_geoph/geoph.1993301819

Hernández Navarro, M.L. (1995). El riesgo de helada en las plantaciones de frutales. El valle medio del Ebro. Institución "Fernando el Católico", Diputación Provincial de Zaragoza, 237 p. 
INE. Instituto Nacional de Estadística (2020). España en cifras. Recuperado el 19 de febrero de 2021 de https://www.ine.es/prodyser/espa_cifras

Jiménez-Donaire, M.P., Giráldez, J.V., Vanwalleghem, T. (2020). Impact of Climate Change on Agricultural Droughts in Spain. Water, 12(11), 3214. DOI: https://doi.org/10.3390/w12113214

Jones, G.V., Duff, A.A., Hall, A., Myers, J.W. (2010). Spatial analysis of climate in wine- grape growing regions in the western United States. American Journal of Enology and Viticulture, 61, pp. 313-326.

Maclean, I.M.D., Suggit, A.J., Wilson, R.J., Duffy, J.P., Bennie, J.J. (2017). Fine-scale climate change: modelling spatial variation in biologically meaningful rates of warming. Global Change Biology, 23(1), pp. 256-268. DOI: https://doi.org/10.1111/gcb.13343

Martí, A. (1991). Relaciones Bioclimáticas del trigo en los Monegros. Geographicalia, 28, pp. 145159. DOI: https://doi.org/10.26754/ojs_geoph/geoph.1991281847

Martí, A. (1993). Cálculo del riesgo de adversidades climáticas para los cultivos: los cereales de verano en Monegros. Geographicalia, 30, pp. 259-269. DOI: https://doi.org/10.26754/ojs_ geoph/geoph.1993301822

Peña-Angulo, D., González-Hidalgo, J.C., Sandonis, L., Beguería, S., Tomás-Burguera, M., LópezBustins, J.A., Lemús-Cánovas, M., Martin-Vide, J. (2021). Seasonal temperature trends on the Spanish mainland: A secular study (1916-2015). International Journal of Climatology. DOI: https://doi.org/10.1002/joc.7006

Quiroga, S., Iglesias, A. (2009). A comparison of the climate risks of cereal, citrus, grapevine and olive production in Spain. Agricultural Systems, 101(1-2), pp. 91-100. DOI: https://doi.org/10. 1016/j.agsy.2009.03.006

Resco, P., Iglesias, A., Bardají, I., Sotés, V. (2016). Exploring adaptation choices for grapevine regions in Spain. Regional Environmental Change, 16, pp. 979-993 DOI: https://doi.org/10.1007/ s10113-015-0811-4

Robert, M., Thomas, A., Bergez, J-E. (2016). Processes of adaptation in farm decision-making models. A review. Agronomy for Sustainable Development, 36, 64. DOI: https://doi.org/10.1007/ s13593-016-0402-x

Rodrigo Comino, J., Senciales, J.M., González Moreno, J.M. (2014). La necesidad de considerar los riesgos climáticos en la introducción de cultivos tropicales en latitudes medias. El mango en el Valle del Guadalhorce (Málaga). Investigaciones Geográficas, 62, pp. 127-141. DOI: https://doi.org/10.14198/INGEO2014.62.09

Rötter, R.P., Palosuo, T., Kersebaum, K.C., Angulo, C., Bindi, M., Ewert, F., Ferrise, R., Hlavinka, P., Moriondo, M., Nendel, C., Olesen, J.E., Patil, R.H., Ruget, F., Taká, J., Trnka, M. (2012). Simulation of spring barley yield in different climatic zones of Northern and Central Europe: A comparison of nine crop models. Field Crops Research, 133, pp. 23-36. DOI: https://doi.org/ 10.1016/j.fcr.2012.03.016.

Sandonis, L., González-Hidalgo, J.C., Peña-Angulo, D., Beguería, S. (2021). Mean temperature evolution on the Spanish mainland 1916-2015. Climate Research, 82, pp. 177-189. DOI: https://doi. org $/ 10.3354 / \mathrm{cr} 01627$ 
Serrano-Notivoli, R., Beguería, S., Saz, M. Á., Longares, L. A., and de Luis, M. (2017). SPREAD: a high-resolution daily gridded precipitation dataset for Spain - an extreme events frequency and intensity overview, Earth System Science Data, 9, pp. 721-738. DOI: https://doi.org/ 10.5194/essd-9-721-2017

Serrano-Notivoli, R., Beguería, S., Saz, M.A., de Luis, M. (2018). Recent trends reveal decreasing intensity of daily precipitation in Spain, International Journal of Climatology, 38(11), pp. 42114224. DOI: https://doi.org/10.1002/joc. 5562

Serrano-Notivoli, R., Beguería, S., de Luis, M. (2019). STEAD: a high-resolution daily gridded temperature dataset for Spain, Earth System Science Data, 11, pp. 1171-1188. DOI: https:// doi.org/10.5194/essd-11-1171-2019

Serrano-Notivoli, R., Tomás-Burguera, M., Martí, A., Beguería, S. (2020). An integrated package to evaluate climatic suitability for agriculture. Computers and Electronics in Agriculture, 176, 105473. DOI: https://doi.org/10.1016/j.compag.2020.105473

Tabuenca, M.C. (1983). Necesidades de frío invernal de variedades de cerezo. En: I Congreso Nacional de la Sociedad Española de Ciencias Hortícolas (Valencia). Comunicaciones SECH, (pp. 661-667).

Tomas-Burguera, M., Vicente-Serrano, S.M., Beguería, S., Reig, F., Latorre, B. (2019). Reference crop evapotranspiration database in spain (1961-2014). Earth System Science Data, 11, pp. 1917-1930. DOI: https://doi.org/10.5194/essd-11-1917-2019

Trnka, M., Rötter, R., Ruiz-Ramos, M., Kersebaum, K.C, Olesen J.E., Zalud, Z., Semenov, M.A. (2014). Adverse weather conditions for European wheat production will become more frequent with climate change. Nature Climate Change, 4, pp. 637-643. DOI: https://doi.org/10.1038/nclimate2242 